\title{
Las ciencias de la vida dentro del BAAS: entre el naturalismo y la teología natural*
}

The Sciences of Life at the BAAS: Between Naturalism and Natural Theology

Juan Manuel Rodríguez Caso ${ }^{\dagger}$

\begin{abstract}
Resumen
La práctica de las disciplinas científicas conlleva una postura metafísica, no siempre explícita. Tradicionalmente, se acepta que la ciencia es netamente materialista, aunque tal aceptación no deja claro si abarca lo filosófico o solamente lo metodológico. Este trabajo plantea la necesidad de recuperar el pluralismo metafísico para poder entender la diversidad de la práctica de la ciencia, tanto desde una perspectiva filosófica como histórica. Se tomará el ejemplo de la institucionalización de la biología en el siglo XIX en el ámbito de las reuniones anuales de la British Association for the Advancement of Science (BAAS). En concreto, la discusión se centrará en analizar el naturalismo científico y la teología natural, como una extensión del debate entre materialismo e idealismo, así como la importancia de esas posturas en la consolidación de la biología victoriana.
\end{abstract}

Palabras clave: Biología - metafísica - teología natural - naturalismo científico - BAAS

\begin{abstract}
The practice of science implies a metaphysical position, not always explicit. Traditionally, it is accepted that science is purely materialistic, although it is not clear if such acceptance covers both the philosophical and the methodological stances. This paper relies on metaphysical pluralism to understand the diversity of scientific practice from a philosophical as well as an historical perspective. To achieve this goal, this article examines the case of the institutionalization of biology in the nineteenth century in the context of the annual meetings of the British Association for the Advancement of Science (BAAS). Specifically, the discussion focuses on analyzing scientific naturalism and natural theology as extensions of the debate between materialism and idealism. Furthermore, it highlights the importance of these positions in the consolidation of Victorian biology.
\end{abstract}

Keywords: Biology - metaphysics - natural theology - scientific naturalis - BAAS

\footnotetext{
* Recibido: 3 de Mayo de 2014. Aceptado en versión revisada: 13 de Julio de 2014.

† Becario Posdoctoral CONACYT, Posgrado en Filosofía de la Ciencia, UNAM, México. Para contactar al autor, por favor, escribir a: juan_manuel_rodriguez_caso@yahoo.es.

Metatheoria 5(1)(2014): 161-173. ISSN 1853-2322.

(C) Editorial de la Universidad Nacional de Tres de Febrero. Publicado en la República Argentina.
} 


\section{Introducción}

Una parte importante de cualquier práctica científica es su institucionalización, ya que a partir de ese momento se da la consolidación de esa práctica de manera consensada por un grupo de científicos. La problemática surge al momento de describir y determinar el momento en que se da tal institucionalización (Tomkins 1963, Lemaine et al. 1976, pp. 13-14, Schaffer 2013, pp. 57-58). Para el caso de las ciencias de la vida, son varias las disciplinas que vienen a la mente, y en este caso nos referiremos al desarrollo de la biología en el contexto de una de las sociedades científicas británicas más influyentes, la British Association for the Advancement of Science (BAAS) durante el siglo XIX. A su vez, esa institucionalización tiene que ver con la unión de la práctica científica específica con una postura metafísica, esta última el sustento filosófico a partir de la cual el científico defiende su visión del mundo (Dupré 1993, pp. 1-2).

La ciencia británica a lo largo del siglo XIX se desarrolló mayormente alrededor de una visión metodológica y filosófica concreta, la teología natural, que puede ligarse con el idealismo. Esta visión combinaba una metodología científica en la que la observación y la contrastación empírica eran la base, a partir de una concepción de la naturaleza en la que ésta se consideraba un reflejo de la obra del Creador. Sin embargo, había otras perspectivas, como la materialista ejemplificada en la propuesta evolutiva del naturalista francés Jean Baptiste Lamarck, que llegaron principalmente desde la Europa continental (Desmond 1989). En ésta, la ciencia se asumía materialista tanto en lo metodológico como en lo ontológico. Ambas posturas ontológicas se mezclaron con la práctica científica británica, de tal manera que como veremos en este trabajo, en términos generales el materialismo se consolidó como la base filosófica del naturalismo científico, mientras que el idealismo jugó un papel similar con la teología natural.

Ahora bien, desde inicios de ese siglo, en Inglaterra no existían de manera explícita disciplinas como la biología, pero sí existían prácticas y teorías que se enfocaban en el estudio de diversos aspectos de la vida, incluido el ser humano. Esos estudios fueron llevados a cabo por individuos que comúnmente se involucraban en sociedades científicas en las que se compartía ese interés común. BAAS fue un foro fundamental para la conformación de la comunidad científica británica, y que desde su fundación en 1831 definió los límites entre las diferentes prácticas científicas, a partir de reunir a los más connotados hombres de ciencia de la época, tanto británicos como extranjeros. Uno de los objetivos que tuvo la Asociación desde su fundación fue la presentación de las diversas áreas de la ciencia al público, que representarían las diferentes prácticas de la ciencia. Esa representación fue un reflejo de la visión particular que los miembros de BAAS tenían sobre la ciencia, y no necesariamente la que existía entre toda la comunidad científica británica.

Desde su fundación, las disciplinas relacionadas con el estudio de la vida fueron relegadas a subsecciones, en virtud que la mayor importancia se dio a las ciencias físicas y químicas al momento de conformar las secciones, debido a que desde el siglo XVI la ciencia británica se había enfocado en los fenómenos físicos, particularmente gracias a trabajos como los de Isaac Newton (Fara 2009, pp. 164 171). A diferencia de estas últimas, para las que no hubo mayor discusión en cuanto a sus alcances explicativos, las ciencias de la vida estuvieron en el foco de la disputa desde un principio.

Cuando inició la Asociación no existía la biología como una disciplina única, sino que había disciplinas específicas como la botánica, la zoología, la anatomía y la fisiología. Se dio también la situación en la que la medicina o la frenología no fueron consideradas disciplinas científicas, aunque la decisión de no incluirlas como parte de la Asociación como veremos tuvo que ver con los alcances políticos e ideológicos de esas propuestas. En cada uno de los casos, la aceptación de una disciplina como parte de la visión de la ciencia que proponía la Asociación estaba ligada a profundas discusiones epistémicas, en las que no solo se incluían aspectos relacionados exclusivamente con la práctica científica, sino también con aspectos sociales y políticos, que influían finalmente en el consenso sobre de las prácticas "adecuadas" para cada disciplina, así como con su aceptación dentro de la comunidad. 
Ese proceso de institucionalización partió del marco del denominado naturalismo científico, una visión que planteaba que el universo y sus características podían ser investigados y entendidos enteramente en términos naturalistas y sobre todo materialistas (Turner 1974, Lightman 1997).

A partir de lo anterior, este ensayo se centrará en explicar la importancia de las posturas metafísicas como parte esencial del desarrollo de las ciencias, más allá de las propuestas sociológicas sobre la construcción de las disciplinas científicas. Las discusiones que se dieron en el ámbito de las reuniones anuales de BAAS durante el siglo XIX muestran los límites dinámicos entre las prácticas científicas, que resultaron en su institucionalización dentro de la comunidad científica británica, y este último proceso se explicará a partir de las diferencias epistémicas entre el naturalismo científico y la teología natural, con énfasis en los aspectos filosóficos y metodológicos subyacentes en cada propuesta. Vale la pena señalar que la disputa entre ambas posturas epistémicas definió el rumbo de la ciencia británica. Considerando trabajos como los de David Cahan y Martin Daunton se puede mostrar que los procesos por los que surgen formalmente las disciplinas no son procesos terminados ni definitivos, sino que las disciplinas científicas están en permanente movimiento, a partir de influencias tanto estrictamente científicas como externas. Es también importante resaltar el papel de la popularización y la difusión en la consolidación del estatus social e intelectual de una disciplina, a partir de la adopción de las prácticas que son comunes a un grupo (Daunton 2005, p. 13). Sin embargo, será a partir del trabajo de John Dupré sobre los límites de las disciplinas científicas que se retomará una postura crítica tanto sobre la "unidad" de la ciencia como el papel de la metafísica en la ciencia, para con ello poder plantear una visión diferente sobre la manera en la que se conforma la práctica de la ciencia.

El trabajo se dividirá en tres secciones: primero, se presentará brevemente la propuesta pluralista de Dupré, para entender las críticas a la idea de concebir la ciencia como una unidad tanto metodológica como ontológica, y en ese sentido aclarar los conceptos a utilizar como materialismo, idealismo y naturalismo. Después, cómo se relacionan estos últimos conceptos con una realidad histórica concreta, como las reuniones de BAAS en el siglo XIX, en donde se enfrentaron posturas como la teología natural y el naturalismo científico, todo esto a partir de la propuesta de las "geografías del conocimiento científico". Finalmente, se presentará el caso de la biología, como un ejemplo de la falta de consenso inicial y del lugar de las ciencias de la vida en general dentro de la Asociación, que resultó en un escaso protagonismo, a expensas de las discusiones que ya había en Europa sobre la importancia de la biología como un campo de conocimiento unificador. Con esto, se busca presentar una visión general de la manera en la que se organizaba el conocimiento científico dentro de un foro como BAAS, que se consolidó como un espacio en el que se reflejaban las diferentes prácticas científicas, en las que a partir de marcos explicativos alternativos, como la teología natural y el naturalismo científico, se buscaba legitimar a la ciencia.

\section{Ciencia y metafísica: entre el materialismo y el idealismo}

La metafísica es el estudio de la naturaleza de los seres y del mundo, y si nos referimos a la ciencia, estamos hablando de la postura a partir de la cual se concibe el mundo que nos rodea, o en otras palaras, entender la naturaleza de la realidad. Aunque como bien lo señalan Peter van Inwagen y Meghan Sullivan, realmente no es fácil decir qué es la metafísica (van Inwagen \& Sullivan 2015), que desde tiempo atrás primero se consideró como el estudio de las causas primeras, las cosas en estado estático, o las categorías de ser y de universal. Dentro de la amplitud de formas de entender la metafísica, aquí se retomará el papel de las presuposiciones metafísicas dentro de la práctica científica. A partir de esto, el punto que se va a considerar es el de la unidad de la ciencia, en el entendido de que las diversas prácticas científicas tienen en común tanto metodología, como epistemología y ontología, una tradición que se ha sustentado desde los tiempos de la antigua Grecia hasta los inicios de la ciencia moderna occidental (Cat 2014). Esta tradición se vio fuertemente reforzada con los trabajos del empirismo lógico, que adoptaron la visión de la "unidad de la ciencia sin metafísica", a partir de la cual se establecía que la práctica científica se unificaba tanto en método como en lenguaje, lo mismo las 
ciencias naturales que las sociales. Vale la pena recordar que al hablar de esa unidad de la ciencia es la física la que normalmente se asumió como la ciencia por excelencia.

Ahora bien, como lo señala Jordi Cat, a partir de un contexto histórico hay que reconocer que hablar de una "unidad" es problemático, ya que hay una variedad de "unidades", sobre todo si consideramos que tal "unidad" puede darse a varios niveles y no necesariamente con la misma fuerza: externa, interna o conectiva, reductiva, sincrónica, diacrónica, epistemológica, ontológica, horizontal, global, local. Dado el tema de este trabajo, aquí se enfatizará la diferencia entre la unidad ontológica y epistemológica, ya que también la metodología se inserta aquí. La unidad ontológica se entiende como la que involucra relaciones entre elementos conceptuales, y esos conceptos pueden describir entidades, propiedades o relaciones y los modelos que se enfocarán a su vez en los aspectos metodológicos de esas conexiones unificadoras. Por otro lado, la unidad epistemológica se refiere a las relaciones como la explicación, y son las conexiones metodológicas y los modelos formales los que pertenecen a este tipo (Cat 2014).

Dupré señala que probablemente la fuente más poderosa para la idea de la ciencia como un proyecto unificado es el compromiso con el monismo, es decir, la creencia de que en un sentido importante, "el mundo está compuesto por algún tipo de cosa" (Dupré 1993, p. 87). Por otro lado, es a partir de su propia consideración que al día de hoy se puede hablar de unos cuantos idealistas dentro de la comunidad científica, es el materialismo la postura metafísica dominante (Dupré 1993, p. 89).

En este punto, vale la pena hacer algunas precisiones conceptuales. El materialismo se entiende aquí como la visión que nada existe excepto el espacio-tiempo, los objetos materiales y los eventos en el espacio-tiempo, y las propiedades ejemplificadas por el espacio-tiempo y los eventos que suceden en esas instancias. Las categorías de objeto material y evento se entienden de manera amplia para incluir cosas como electrones y campos, y al mismo tiempo excluyen de manera explícita cosas como Dios, almas, ángeles y conceptos relacionados (Rea 2002, p. 25). Como lo señaló en su momento Armstrong, E. Russell señalaba que el materialismo era aceptable como "una máxima práctica del método científico" (Armstrong 1973, p. 62), una idea apoyada fuertemente desde tiempo atrás por Thomas H. Huxley a partir de la afirmación de la primacía del materialismo tanto metodológico como filosófico en la ciencia (Huxley 1869).

Por otro lado, el idealismo se entiende como la postura de que la realidad, y el conocimiento que podemos obtener de la misma es construido en nuestra mente, y por lo tanto, no es material; en una postura más extrema se asume que todos los seres están compuestos por mente o espíritu. Esto nos lleva a la dicotomía entre los dos extremos sobre la concepción de la realidad, ejemplificada en el dualismo cartesiano. Hay un tercer concepto en el que conviene detenerse, el naturalismo. Éste se puede dividir en ontológico y en metodológico, en donde el primero se refiere al estudio filosófico de la naturaleza de la realidad, razón por la que se puede equiparar con el materialismo. El segundo se refiere a la práctica científica, esto es, la comprensión del mundo a través de hipótesis, teorías, explicaciones, deben darse a través del método científico (experimentos, leyes naturales), sin que ello implique necesariamente un compromiso ontológico (Mander 2011, pp. 4-6). A partir de estas definiciones, el naturalismo ontológico puede considerarse como un sinónimo del materialismo, al ser explícitos ambos en su rechazo al idealismo, aunque podemos señalar que la diferencia entre ambos conceptos es que el materialismo es una postura filosófica como tal, mientras que el naturalismo es una aproximación general a la concepción del mundo. Estas definiciones, u otras similares, se aplican actualmente para definir las diferentes posturas epistémicas, y que como veremos en las siguientes secciones, pueden ser utilizadas en el mismo sentido para el análisis de casos históricos, ya que a pesar del paso del tiempo, con pequeñas diferencias, cada definición mantiene un sentido similar.

Quiero ser cuidadoso con estas diferencias, ya que es común que en las discusiones filosóficas y científicas contemporáneas sobre estos conceptos se suele caer en los extremismos al asumir que como individuo -ya sea un filósofo o un científico por ejemplo- se debe de retomar exclusivamente una postura metafísica y defenderla hasta las últimas consecuencias. De manera breve, podemos señalar el ejemplo del llamado conflicto entre evolucionismo y creacionismo, particularmente de la situación que se ha vivido en Estados Unidos desde inicios del siglo XX, en la que los extremos han dominado la 
discusión, el materialismo del proceso evolutivo contra el idealismo del creacionismo. Sin embargo, tenemos ejemplos como el de Reiss que hace hincapié en el papel de la personalidad como un factor fundamental en la manera en la que cada individuo prioriza tanto el conocimiento como las creencias, con lo que individuos que pudieron tener la misma formación académica pueden perfectamente defender posturas diferentes (Reiss 2005).

Aquí se plantea una propuesta en la que a partir del discurso filosófico se puede reinterpretar la historia de la ciencia. Charles S. Myers a inicios del siglo XX ya dejaba ver las complicaciones que implicaba favorecer exclusivamente una u otra postura, para lo que resaltaba la necesidad de contemplar igualmente válidos cualquier forma de monismo o dualismo (Myers 1901, pp. 475-476). Al retomar la postura de Dupré sobre el pluralismo como una opción filosófica para justificar la metafísica, es que por un lado se hace hincapié en la no unidad de la ciencia, y por el otro se abre la posibilidad a que desde un punto de vista histórico, se plantee una visión alterna sobre el papel de las posturas metafísicas en la construcción y práctica de las disciplinas científicas, en este caso la biología, que es "seguramente la ciencia que aborda mucho de lo que es de gran preocupación para nosotros seres biológicos" (Dupré 1993, p. 3).

En la siguiente sección, me enfocaré en presentar esa pluralidad metafísica existente dentro de una comunidad de individuos en un lugar y momento específico, la Inglaterra victoriana de mediados del siglo XIX.

\section{La ciencia británica desde la visión de BAAS: el naturalismo materialista vs. la teología idealista}

La ciencia británica fue dominada durante varios siglos por la élite intelectual, concentrada especialmente en los principales enclaves educativos, controlados por los anglicanos conservadores, como Oxford y Cambridge, así como las sociedades científicas concentradas en Londres, como la famosa Royal Society, en donde la visión de la ciencia estaba ligada a la teología natural, y en ese sentido, a una forma de naturalismo idealista. Hasta inicios del siglo XIX, las ciencias de la vida en general habían sido relegadas a un segundo plano por las ciencias físicas. La filosofía natural y posteriormente la historia natural aunque desarrolladas e impulsadas por personajes tan influyentes como Francis Bacon (1561-1626) y Robert Boyle (1627-1691) habían estado sujetas al protagonismo de la física y de las matemáticas.

Los planteamientos de Bacon y posteriormente de William Whewell establecieron la base de lo que sería la práctica apropiada de la ciencia en un sentido amplio, al considerar que desde la filosofía natural se podía explicar la teología natural, todo ello dentro de un marco idealista (Yeo 1979, pp. 495498), que fue la visión dominante en la filosofía durante la mayor parte del siglo XIX (Collini 1978, p. 4). De hecho, la aportación de Whewell puede verse como definitiva, porque aunque resulte redundante, la palabra "científico" con la cual se designa a aquel que practica alguna actividad relacionada con la ciencia fue acuñada en 1833 (Yeo 2003, p. 5), pero siempre dentro del contexto de la teología natural. Esto se reafirma a partir de la intención con la que se funda la Asociación, que fue la de presentar una visión unificada de la ciencia dentro del contexto de la teología natural. Aunque es cierto que eso se dio con ciertos rasgos liberales, lo cual en un principio no significó una aceptación del materialismo como opción alternativa, ya que éste se relacionó durante los primeros años del siglo XIX con la Revolución Francesa, y por lo tanto, con inestabilidad social.

Con el paso de los años, esta intención de enfatizar la unidad metodológica de la ciencia se fue debilitando en favor de una visión en la línea de lo planteado por Whewell, en la que se enfatizaban las diferentes ramas de la ciencia pero sin caer en la discusión inicial de su posible unidad. Autores como David Cahan plantean que más que hablar de la ciencia o de disciplinas científicas, de lo que se habla es del conocimiento y de cómo se organizaba a partir de los consensos que podían surgir entre grupos de individuos que compartían prácticas similares (Cahan 2003, p. 293). Aquí se tiene que hacer la diferencia entre el consenso social que podía derivar en hablar de una disciplina científica consolidada, 
y de la naturaleza propia de cada práctica científica. Como veremos más adelante con la biología, cada campo de conocimiento estaba conformado por metodologías muy diferentes en las que confluían también posturas filosóficas diferentes.

Ahora bien, como se mencionaba al principio, la fundación de la Asociación estuvo motivada por la búsqueda de una forma diferente de hacer ciencia, a la establecida por Oxbridge. La corriente dominante, la teología natural, había impuesto una visión de la ciencia en la que la metodología científica era la que la búsqueda última del Creador era fundamental, siempre a través de la razón y no de la revelación (Morrell \& Thackray 1981, pp. 36-46, 224-229), una postura metodológica naturalista con una fuerte influencia idealista. Pero esa búsqueda tenía límites, que para muchos filósofos e historiadores naturales a inicios del siglo XIX empezaron a resultar problemáticos. La alternativa que se fue consolidando fue el naturalismo científico, con un componente materialista, una postura que ya había avanzado con Bacon, y que ante todo buscaba eliminar las explicaciones sobrenaturales de los fenómenos naturales.

Gran Bretaña se encontraba en un momento socialmente complejo a inicios del siglo XIX, y la ciencia no era ajena a esa complejidad. La educación solo estaba al alcance de la élite, tanto en términos políticos como económicos, y la consolidación del capitalismo trajo consigo la ampliación de las brechas sociales. El "radicalismo" hizo presencia sobre todo entre las clases menos favorecidas, en la búsqueda de opciones políticas, educativas y sociales frente al conservadurismo tradicional. En el plano de la educación, la aparición de universidades como la de Londres y Edimburgo dio opciones a las nuevas generaciones, al plantear que el estudio de la ciencia no debía estar sujeto a los dictados de la teología natural, y que no solamente los anglicanos podían tener acceso a educación de calidad. Fue así como en el primer cuarto de siglo fue cada vez más notoria la necesidad de foros abiertos al público que promovieran la ciencia, por lo menos una visión con rasgos cada vez más claramente materialistas, resultado de la influencia del transformismo lamarckista francés (Desmond 1989, pp. 1-24).

El BAAS es importante para la ciencia británica porque se convirtió rápidamente en un foro legitimador para las diversas prácticas científicas, y mucho de ese éxito se debió a que las reuniones anuales permitieron realzar la importancia de la ciencia como parte del avance de la nueva sociedad capitalista e industrial. En este sentido, no hay que olvidar que los llamados "caballeros de la ciencia" (gentlemen of science) que fundaron la Asociación no eran "profesionales" de la ciencia en sentido estricto, sino que eran hombres de posibilidades económicas que dedicaban parte de su tiempo a la práctica científica, siempre dentro de un contexto fuertemente idealista. Como lo han señalado Jack Morrell y Arnold Thackray en su trabajo clásico sobre los inicios de la Asociación, fue la visión establecida por hombres como David Brewster, William V. Harcourt y John Phillips, que eran aristócratas con intereses científicos, para dar lugar a un foro alternativo para la ciencia, que la llevara y la difundiera a lo largo y ancho de la provincia británica (Orange 1972, Morrell \& Thackray 1981, pp. 36-94).

Aunque hay que tener claro que el plantear un foro alternativo para la ciencia no significó un cambio radical ni mucho menos. BAAS fue un foro que buscó diferenciarse de la teología natural dominada por el anglicanismo tradicional de Oxbridge, lo que abrió las puertas a anglicanos liberales y no-conformistas (Non-conformists), pero al mismo tiempo cerró las puertas en un inicio a judíos, católicos, metodistas, bautistas, materialistas y ateos. Con esto, se planteó como un foro neutral, y aunque en teoría se planteaba una separación entre la ciencia y la religión, en la práctica los participantes insistían en mantener una congruencia entre la religión natural y la revelación. Lo anterior da una idea de la importancia de la teología natural, y por lo tanto del idealismo, como parte de la ciencia británica, y al mismo tiempo de la diversidad de posturas que existían dentro de la tradición británica, un punto reafirmado por Topham. Desde un inicio se planteó la necesidad de que el avance de la ciencia debía darse a partir de una separación entre la práctica científica y otras facetas culturales, pero con el paso de los años fue notorio que el peso del marco teórico de la teología natural pesó mucho.

Pero como se señaló al principio de esta sección, la Asociación buscó establecer "su" definición de ciencia, y lo hizo dentro de un marco de explicación concreto, que fue la teología natural (Morrell \& 
Thackray 1981, pp. 224-297). Ha sido común en la historiografía de la ciencia que la imagen que se tiene de los escritores de esta corriente durante siglo XIX para explicar la naturaleza ha partido de suponer un compromiso con uno de los argumentos clásicos de la teología natural, el argumento del diseño, que parte de la existencia de un diseñador divino del que se infiere un diseño aparente en la naturaleza. Sin embargo, como lo señala Jonathan R. Topham, la teología natural no era solamente eso, sino una búsqueda general por racionalizar la revelación cristiana para así comprender la Creación (Topham 2010, p. 60).

Con esto, lo que se pretende es hacer ver la complejidad de la labor científica que se dio en un espacio como BAAS, ya que la diversidad de prácticas de los hombres de ciencia estaba circunscrita además a una gran variedad de posiciones teológicas y filosóficas. Ahora bien, este ambiente de discusión se mantuvo en términos generales dentro de la Asociación hasta inicios de la década de 1850. El trabajo clásico de Turner muestra cómo a partir de esa época empieza un cambio dentro de la comunidad científica británica, que va de la mano a la aparición de una nueva generación de científicos, formados ya no en las tradiciones de Oxbridge, sino en universidades como Londres y Edimburgo, con metodologías y filosofías cercanas a las tradiciones científicas de Francia y Alemania, que como se mencionó anteriormente no funcionaban dentro del marco de la teología natural (Turner 1974). De hecho, fue en esos ambientes "radicales" en donde empezó a consolidarse una visión alternativa de la ciencia, fundamentada en la existencia de leyes naturales cuyo funcionamiento no apelaba a un papel activo del Creador, sino que incluso no lo consideraba como una parte esencial de la explicación. Es decir, un claro llamado al materialismo como la explicación científica tanto en términos metodológicos como ontológicos. Esta posición, conocida como naturalismo científico, ganó adeptos rápidamente entre las nuevas generaciones de hombres de ciencia, sobre todo en aquellos que buscaban hacer de la ciencia una profesión (Lightman \& Dawson 2014). Personajes como Huxley, John Tyndall y John Lubbock fueron ejemplo de esos personajes, que con el paso de los años se ubicaron en posiciones políticas relevantes que les permitieron colocar a la ciencia como un aspecto preponderante de la cultura, incluso mucho más que la religión, aunque esta última postura no era sostenida con igual intensidad por todos los naturalistas científicos (Barton 1998, Lightman \& Dawson 2014), una situación que de nuevo lleva a considerar la diversidad de posturas filosóficas presentes en las discusiones científicas.

El avance del naturalismo científico dentro de la Asociación fue notorio, sobre todo si se considera la disminución en el protagonismo de los clérigos y religiosos en general, tanto en el funcionamiento burocrático como en las presentaciones académicas. Ciaran Toal ha reafirmado esta idea al señalar el avance del secularismo dentro de la Asociación a partir de la década de 1870. Aunque hay que ser cautelosos con esta afirmación, ya que aunque la disminución de clérigos fue notoria, la presencia de la religión en general se mantuvo, y eso repercutió directamente en la manera en la que se concebía la práctica de la ciencia (Toal 2012). A pesar de la disminución de clérigos, las posturas materialistas ganaron espacios tanto dentro de BAAS como en otras asociaciones científicas, pero no al punto de sustituir la teología natural, sino que se dio un proceso en el que la gama de posturas se amplió notablemente.

A lo largo del siglo hubo numerosos cambios en la organización interna de la Asociación, con un notable incremento en el número de secciones, que buscaba representar la diversidad de prácticas, pero las posturas filosóficas no eran necesariamente discutidas, o por lo menos había una apertura que permitía su presencia en las reuniones. Recordar aquí que los miembros de la Asociación reconocían a los campos de conocimiento más consolidados a partir de buscar un equilibrio entre los intereses políticos de la Asociación con las posturas particulares de los miembros más connotados. No obstante, es cierto que los cambios por los que surgía una sección o un cambio de nombre, eran el resultado de una larga y compleja discusión entre los "caballeros de la ciencia", en la que se mezclaban tanto intereses políticos como puntos de vista filosóficos a partir de los cuales se definía el rumbo de la ciencia británica, tanto en general como en particular, por lo menos desde la perspectiva de BAAS.

Como se mencionó al principio, la disputa entre la teología natural y el naturalismo científico, sobre todo a partir de finales de la década de 1860 , definieron el nuevo rumbo de la ciencia británica. 
Aunque como ya se mencionó anteriormente, la teología natural no desapareció con la irrupción del naturalismo, sino que ambas posturas se mantuvieron vigentes dentro de la práctica científica.

\section{Biología: la búsqueda de una identidad institucional}

Como lo ha señalado de manera acertada Joseph A. Caron en su detallada reconstrucción historiográfica sobre la historia de la biología, debemos ser cuidadosos al ver que no hubo una sola tradición sobre la que se desarrolló la biología como disciplina. Al contrario, en Europa se deben ubicar por lo menos tres tradiciones que plantearon de manera particular qué era la "biología". Caron afirma que a lo largo de la historia se han propuesto muchos candidatos como el posible fundador de la biología, y con cada uno "es asociado un concepto particular o algún avance extraordinario en lo metodológico o en lo teórico como la señal de la creación de conocimientos rigurosamente científicos de los procesos vitales" (Caron 1988, p. 224). Esto último es posiblemente uno de los retos (o uno de los impedimentos como se menciona más adelante) por los que la historia de la biología se ha centrado en algunas tradiciones, de las que podemos reconocer por lo menos tres que marcaron el devenir no solo de la biología, sino de la ciencia en Europa: Alemania, Francia e Inglaterra.

Lo anterior nos da una idea de la complejidad que reviste la idea de reconstruir el desarrollo e institucionalización de una disciplina como la biología si lo vemos desde una perspectiva global en la que se conjuntaron factores divergentes. Entre esos factores estuvieron enfoques metodológicos diferentes sobre la manera apropiada de acercarse al estudio de la vida (ejem. énfasis en la anatomía, o resaltar las relaciones históricas entre los individuos), todo esto en contextos filosóficos que van desde el idealismo hasta el materialismo, con diferentes matices. En este sentido, como se mencionaba al principio de la sección, el estudio de la vida, en cualquiera de sus aspectos, se había dado desde mucho tiempo atrás. La observación y descripción de plantas y animales en cuanto a sus formas o sus costumbres, la clasificación de la naturaleza para entender el orden divino, entender el funcionamiento de fenómenos fundamentales como la respiración, la digestión o la circulación, todos y cada uno son y han sido aproximaciones que han buscado entender el fenómeno de la vida. Las metodologías han variado desde la disquisición filosófica que buscaba entender a los organismos vivos en un plano ideal, hasta aplicar los principios del mecanicismo de moda de la física para resignificar los fenómenos vitales desde un punto de vista reduccionista. Pero como ya se señalaba anteriormente, pueden ser tantas las metodologías o teorías como autores que podríamos considerar. Es por ello que buscar entender los procesos por lo que se da la consolidación e institucionalización de una disciplina científica se vuelven fundamentales.

Aquí se retomará la metodología de las "geografías del conocimiento científico", para justificar el estudio concreto de una disciplina en un momento y lugar específicos, para con ello comprender el desarrollo local de la ciencia como una manera de entender a su vez el desarrollo global (Livingstone 2003). En el caso británico, de manera breve, las ciencias de la vida siempre jugaron un papel secundario para los hombres de ciencia, ya que como se planteó en la primera sección, la metodología establecida por influyentes personajes como Bacon o Whewell enfatizaban la importancia de la experimentación a la par de aplicar leyes naturales que explicaran los fenómenos de la naturaleza. El ejemplo arquetípico en este sentido es el trabajo de Sir Isaac Newton (1643-1727) sobre la fuerza de gravedad, un enfoque que desde la física fue muy influyente en otras ciencias y en otros contextos. O, como fue el caso de Buffon en Francia, quien fue influenciado por la propuesta newtoniana en su planteamiento sobre el estudio de la historia natural, al reivindicar el método inductivo al querer llegar a conclusiones teóricas a partir de "hechos" (Sloan 1987, pp. 108-109).

Las ciencias de la vida en Gran Bretaña se desarrollaron en ámbitos particulares como las sociedades científicas provinciales, en los que las influencias de la metrópoli o del continente no eran tan fuertes, con lo que visiones particulares surgieron con el paso de los años (Alberti 2001). Desde la fundación de la Asociación en 1831, las ciencias de la vida estuvieron presentes, aunque su protagonismo fue relativamente menor hasta por lo menos mediados de la década de 1860 . Por un 
lado, los dirigentes de la Asociación fueron físicos, matemáticos, químicos o geólogos, que normalmente daban mayor cabida a sus propias disciplinas, que además estaban mucho más consolidadas institucionalmente, sobre todo por sociedades con sede en Londres. La zoología, la botánica, la anatomía y la fisiología fueron las secciones en las que se dividieron a las ciencias de la vida, como un ejemplo de la diversidad de prácticas que había, todas bajo una visión idealista; cada una correspondía a una práctica y metodología específica, adecuada a su objeto de estudio, y en ese sentido se consideraban independientes. Fuera de la Asociación, las discusiones sobre la biología fueron escasas. La figura que mayor impulso dio a la unificación de las ciencias de la vida bajo una sola disciplina fue Huxley, particularmente gracias a las presentaciones que realizó a finales entre 1855 y 1858 en la Royal Institution, como parte de las Fullerian Lectures, cuyo objetivo era relacionar la fisiología con la anatomía. Estas presentaciones llevaron por título general "Biology" (Royal Institution of Great Britain 1858), con lo que Huxley buscaba presentar su visión sobre lo que en el resto de Europa, como en Francia o en Alemania ya se discutía desde principios del siglo, y como se mencionaba antes, a partir de los trabajos ya conocidos de personajes como Lamarck (1744-1829) y Gottfried Reinhold Treviranus (1776-1837) (White 2003, pp. 51-58). La diferencia de la propuesta de Huxley con respecto a lo que se discutía en BAAS desde hacía varios años era el énfasis materialista en las explicaciones naturalistas, como el rechazo explícito a las intervenciones sobrenaturales.

Desde que se empezó a utilizar el término, la biología se planteó como un espacio en el que se unificaban las diversas prácticas que desde diversos puntos de vista abarcaban las ciencias de la vida. Como lo señala Robert Richards, ese eje unificador fue desde un inicio la evolución o transformismo, aunque es cierto que esto generó con el paso de los años un sesgo que ha sido difícil de erradicar de la historia de la biología. Richards ha sido muy crítico en cuanto al poco interés que ha habido en considerar la historia de la biología desde una perspectiva mucho más integral, ya que al poner atención solo en un hombre (Charles Darwin), en un libro (El origen de las especies, 1859), y en una teoría (la selección natural), se han dejado de lado las aportaciones de otras áreas (ecología, embriología, bacteriología, por mencionar solo algunas) u otros personajes y teorías, que sin lugar a dudas enriquecen a la biología en su práctica (Richards 2003).

Como se mencionaba brevemente antes, la concepción de Huxley sobre la biología buscaba ser un cambio radical con respecto a la tradición idealista impuesta por la historia natural, ya que ponía énfasis en que la práctica debía darse dentro de un laboratorio, y no en el campo, con lo que "el laboratorio, más que el campo, se volvió el sitio para estudiar la nueva Naturaleza” (Desmond 1999, p. 419), una idea que buscaba enfatizar el aspecto material de las explicaciones científicas. La historia natural británica había estado dominada por una práctica descriptiva de los fenómenos de la naturaleza, en la línea de la tradición de la teología natural. Un claro ejemplo de ese tipo de práctica fue el anatomista Richard Owen (1804-1892), quien durante la primera mitad del siglo XIX fue considerado como el más importante expositor de esta práctica de las ciencias de la vida. De hecho, la disputa más importante que se dio sobre cuál era la práctica adecuada para las ciencias de la vida sucedió entre Huxley y Owen, una situación que se dio tanto fuera como dentro de la Asociación. Los enfrentamientos entre ambos personajes tuvieron su momento más álgido a principios de la década de 1860, cuando a lo largo de tres reuniones consecutivas (1860 en Oxford, 1861 en Manchester, y 1862 en Cambridge) se enfrentaron en diferentes discusiones sobre un tema concreto que fue el origen y desarrollo del hipocampo. El punto de partida de ambos fue explicar desde un punto de vista natural al ser humano, y para ello se valieron ambos de sus amplios conocimientos en anatomía y zoología. La diferencia surgió al momento de concebir el lugar del hombre en la naturaleza: Owen lo consideraba como algo único, que no podía ser explicado sino mediante una explicación religiosa, mientras que Huxley concebía que debía ser explicado en su totalidad por la ciencia. Ambos autores partían de una metodología que analizaba detalladamente la anatomía del hombre, incluso al resaltar su relación con otros organismos como los chimpancés y los gorilas, pero cada uno tenía un marco general de explicación diferente. La teología natural que influía el trabajo de Owen implicaba límites en la explicación, como el caso del origen del ser humano, mientras que el naturalismo que impulsaba 
Huxley buscaba romper esos límites al considerar que mediante las leyes naturales era posible explicar cualquier cosa (Rupke 2009, pp. 192-204).

Como se verá también en la siguiente sección, 1866 fue un año definitivo para la consolidación de las ciencias de la vida dentro de la Asociación. A instancias de Huxley, quien ya llevaba años promoviendo la importancia de la biología como una ciencia que podía unificar a las diferentes disciplinas enfocadas en el estudio de diversos aspectos de la vida en el planeta, logró el consenso de un grupo importante de jóvenes e influyentes científicos, como el caso del arqueólogo John Lubbock (1834-1913) y del físico John Tyndall (1820-1893) para apoyar su llegada a la presidencia de la Sección D, tradicionalmente conocida como Zoología y Botánica. Ese grupo, conocido como el "Club X", se consolidaría como la base del profesionalismo de la ciencia a partir de ese momento (Barton 1998). El empuje de Huxley logró que se le cambiara el nombre a la sección, para finalmente ser denominada Biología, y en ella incluir al resto de disciplinas como la zoología, la botánica, la anatomía, la fisiología, y también por primera vez, la antropología. Pero no solamente logró que se cambiara el hombre, sino que logró de manera explícita que la biología fuera reconocida como la práctica que podía unificar al conjunto de las ciencias de la vida (The Athenaeum, Sept. 1, 1866, p. 277).

Años después, en 1876, la presidencia de la sección recayó en Wallace, quien en su discurso presidencial dejaba ver la amplitud de la disciplina, al considerar las diversas prácticas incluidas en la misma: anatomía, fisiología, embriología, histología, clasificación, evolución (Report 1877, p. 100). Entre los detalles más interesantes que destaca Wallace es la relación entre los organismos vivos con su ambiente, y pone énfasis en el papel de la biología como la disciplina que permitiría comprender el origen y progreso del hombre. Contrario a lo que muchos científicos podían pensar sobre los avances de la ciencia, Wallace mencionaba que a pesar de los avances en temas como el origen de la vida y la antigüedad del hombre no podía hablarse de cambios radicales aunque hacía particular énfasis en resaltar la importancia de la biología para explicar esos puntos (Report 1877, pp. 118-119).

Esta visión unificada de las ciencias de la vida desde un punto de vista metodológico se impuso dentro de la Asociación. En particular, la sección D se consolidó alrededor de esa visión que fue promovida tanto fuera como dentro de BAAS por Huxley. En ésta, la biología lograba unificar los criterios sobre los que habría de estudiarse la vida, aunque esta situación se mantuvo solamente hasta 1894. A partir de la siguiente reunión, en 1895, las discusiones entre los zoólogos, botánicos y fisiólogos derivaron en una nueva separación de las disciplinas. La sección D se renombraría como Zoología, para la fisiología se abriría la sección I (que incluía tanto patología como psicología experimental), y para la botánica se crearía la Sección K (Report 1900), dando con ello un vuelco a la propuesta de unificar las ciencias de la vida. Esta situación se dio en parte por la amplitud de la investigación que había para esa época en cada una de las disciplinas, ya que no se ponía en duda que la biología fuera el campo de conocimiento que unificaba a las demás a partir de considerar las diversas facetas del estudio de la vida.

Recapitulando brevemente, la postura de Huxley retomó las diversas metodologías para unificarlas alrededor de una postura metafísica concreta como fue el materialismo. Lo que logró en BAAS tuvo repercusiones en un ámbito más general, ya que planteó la necesidad de discutir abiertamente las posturas metafísicas de los científicos, aunque en la línea de los intereses del Club X, Huxley buscaba establecer una visión única de la ciencia. Por lo menos en el caso británico, logró que se diera el reconocimiento al materialismo como una filosofía válida para la ciencia, lo que permitió a muchos biólogos encontrar una alternativa al idealismo defendido por la teología natural.

\section{Conclusiones}

El foro de BAAS fue el espacio en que se legitimó la práctica de la ciencia británica a lo largo del siglo XIX. Aunque desde su fundación en 1831 se planteó como un espacio alternativo a la ciencia "oficial", ejemplificada por la teología natural, con el paso de los años y guiados por los intereses de los "caballeros de la ciencia", su importancia dentro de la comunidad científica británica y para el público 
en general logró dotar a la Asociación de una autoridad capaz de definir los límites de la práctica científica. Esos límites fueron impuestos a partir de una visión concreta de la ciencia, motivada por intereses ideológicos particulares, como fue el concebir a la práctica científica como una extensión de los intereses del Imperio, a pesar de la intención inicial de concebir a la Asociación como un foro políticamente neutral. El planteamiento de Dupré sobre el pluralismo filosófico proporciona un marco adecuado para justificar y entender que la práctica científica va de la mano de posturas metafísicas alternativas. Aunque desde la sociología se podría asumir el consenso en favor de una u otra postura como la adecuada, desde la filosofía se puede ver que visiones como el idealismo o el materialismo tienen cabida, independientemente de la metodología científica.

Las disputas que se dieron dentro de la Asociación al momento de definir cuáles eran disciplinas aceptables para ser consideradas como auténticas ciencias se tiene que ver en un sentido amplio. Disciplinas relacionadas con la física y las matemáticas tenían un reconocimiento amplio, ya que la metodología científica vigente inspirada en las propuestas de filósofos naturales como Bacon, Boyle y Whewell impuso una forma concreta de ver la naturaleza a través de leyes naturales, de resaltar la necesidad de la experimentación y de imponer el mecanicismo para entender los fenómenos (Ellegård 1990, pp. 177-178). A partir de estas propuestas, la ciencia se consolidó como una práctica que buscaba obtener conocimiento de la naturaleza a partir de una metodología diferente a la de la filosofía, pero no por ello se alejó de los objetivos de la teología. La teología natural, entendida como el medio racional para entender la obra divina, se mantuvo como el marco de explicación vigente entre la comunidad británica desde el siglo XVII. Fue hasta mediados del siglo XIX que una alternativa surgió, el naturalismo científico, que planteaba de manera explícita una separación entre la búsqueda y el entendimiento de la naturaleza como medio para entender la obra de Dios y la necesidad de entender esa misma naturaleza sin tener que apelar al Creador. Con esto, la metodología en sí misma no era un punto de discusión, sino las razones que motivaban a cada individuo para realizar la práctica científica desde determinada postura metafísica.

En ambos casos, los límites entre campos de conocimiento con un objetivo común fueron dinámicos, sujetos a negociaciones e intereses de los miembros de la Asociación. Esto permitió que las disciplinas cambiaran con el paso del tiempo, por lo menos en cuanto al nombre que las designaba, ya que las prácticas específicas se mantuvieron vigentes a lo largo del siglo. Si seguimos los planteamientos de David Cahan y Martin J. Daunton, el estatus institucional de disciplinas como la biología se consiguió a partir de la difusión de prácticas que encontraron consenso en un grupo de científicos. Aunque hay que ser cautelosos ya que las disciplinas están en constante cambio, por lo que no se puede hablar de una consolidación definitiva, y mucho menos si consideramos que ese dinamismo está relacionado con factores tan diversos como la cultura y el momento histórico.

A partir de lo presentado en este trabajo, hay que hacer ver la necesidad de un análisis histórico de la filosofía de la biología desde los contextos culturales y temporales concretos en los que se dan las diferentes prácticas científicas, como el caso de los estudios enfocados en las provincias. El marco de la teología natural se mantuvo vigente incluso hasta el siglo XX, aunque es cierto que el naturalismo se impuso como una visión dominante, sobre todo en términos del discurso ideológico de lo que debía ser la ciencia "profesional". La biología, como una disciplina desarrollada en el ámbito de las reuniones de BAAS, es un ejemplo concreto de cómo la práctica científica se institucionalizó alrededor de una pluralidad de posturas metafísicas, como la teología natural y el naturalismo científico.

De cualquier forma, los procesos por los que se institucionalizaron las disciplinas en Gran Bretaña requiere considerar este último punto, ya que no se puede dejar de lado el hecho de que la ciencia no es necesariamente una práctica monolítica, y mucho menos si tomamos en consideración que los científicos son individuos con personalidades independientes, sujetas a motivaciones particulares (Reiss 2005). Aunque puede sonar aventurado, es en los detalles históricos y sociales en donde se podrá tener una comprensión más clara sobre los alcances de la filosofía de la biología. Entender las prácticas científicas desde lo local tiene la ventaja de poder enfocarse en detalles que de otra manera se pueden pasar por alto, aunque claramente es una visión reduccionista que necesita conectarse con la historia global, para así poder comprender el contexto en el cual se ha formado y practicado la ciencia. 
Finalmente, recapitular la historia a partir de discusiones filosóficas como las posturas metafísicas de quienes practican la ciencia, nos debe hacer ver la necesidad de entender las implicaciones de cada postura, para así comprender los alcances y limitaciones, tanto metodológicos como filosóficos de las disciplinas, pero sobre todo, de quienes las ponen en práctica, tanto antes como ahora.

Bibliografía

Alberti, S.J.M.M. (2001), "Amateurs and Professionals in One County: Biology and Natural History in Late Victorian Yorkshire", Journal of the History of Biology 34(1): 115-147.

Armstrong, A.M. (1973), “On Methodological Materialism”, Philosophy and Phenomenological Research 34, $62-72$.

Barton, R. (1998), “'Huxley, Lubbock, and Half a Dozen Others": Professionals and Gentlemen in the Formation of the X Club, 1851-1864", Isis 89(3): 410-444.

Cahan, D. (2003), From Natural Philosophy to the Sciences: Writing the History of Nineteenth-Century Science, Chicago: University of Chicago Press.

Caron, J.A. (1988). “«Biology» in the Life Sciences: A Historiographical Contribution”, History of Science 26: 223-268.

Cat, J. (2014), "The Unity of Science", en Zalta, E.N. (ed.), The Stanford Encyclopedia of Philosophy, URL = http://plato.stanford.edu/archives/win2014/entries/scientific-unity/.

Collini, S. (1978), “Sociology and Idealism in Britain 1880-1920”, European Journal of Sociology 19: 3-50.

Daunton, M.J. (2005), The Organisation of Knowledge in Victorian Britain, Oxford: Oxford University Press.

Desmond, A. (1989), The Politics of Evolution: Morphology, Medicine, and Reform in Radical London, Chicago: University of Chicago Press.

Desmond, A. (1999), Huxley: From Devil's Disciple to Evolution's High Priest, London: Perseus Books.

Dupré, J. (1993), The Disorder of Things: Metaphysical Foundations of the Disunity of Science, Cambridge, Massachusetts, London, England: Harvard University Press.

Ellegård, A. (1990), Darwin and the General Reader: The Reception of Darwin's theory of Evolution in the British Periodical Press, 1859-1872, Chicago: University of Chicago Press.

Fara, P. (2009), Science: A Four Thousand Year History, Oxford: Oxford University Press.

Huxley, T.H. (1869), "On the Physical Basis of Life”, The Fortnightly Review 5: 129-45.

Lemaine, G., Macleod, R., Mulkay M. y P. Weingart (1976), Perspectives on the Emergence of Scientific Disciplines, Paris: Walter de Gruyter.

Lightman, B.V. (ed.). (1997), Victorian Science in Context, Chicago: University of Chicago Press.

Lightman, B.V. y G. Dawson (eds.) (2014), Victorian Scientific Naturalism: Community, Identity, Continuity, Chicago: University of Chicago Press.

Livingstone, D.N. (2003), Putting Science in its Place: Geographies of Scientific Knowledge, Chicago: University of Chicago Press.

Mander, W.J. (2011), British Idealism: A History, Oxford: Oxford University Press.

Morrell, J. y A. Thackray (1981), Gentlemen of Science: Early Years of the British Association for the Advancement of Science, Oxford: Clarendon Press.

Myers, C.S. (1901), "Naturalism and Idealism", The Philosophical Review 10: 463-476.

Orange, A.D. (1972), "The Origins of the British Association for the Advancement of Science", The British Journal for the History of Science 6(2): 152-176.

Rea, M.C. (2002), World Without Design: The Ontological Consequences of Naturalism, Oxford: Clarendon Press.

Reiss, S. (2005), "Human Individuality and the Gap between Science and Religion”, Zygon ${ }^{\circledR}$ 40(1): 131-142. 
Report of the Meeting of the British Association for the Advancement of Science, London: John Murray.

Richards, R. (2003), "Biology", en Cahan, D. (ed.), From Natural Philosophy to the Sciences: Writing the History of Nineteenth-Century Science, Chicago: University of Chicago Press, pp. 19-30.

Royal Institution of Great Britain (1858), Proceedings.

Rupke, N.A. (2009), Richard Owen: Biology without Darwin, Chicago: University of Chicago Press.

Schaffer, S. (2013), "How Disciplines Look", en Barry, A. y G. Born (eds.), Interdisciplinarity: Reconfigurations of the Social and Natural Sciences, New York: Routledge, pp. 57-81

Sloan, P.R. (1987), "From Logical Universals to Historical Individuals: Buffon's Idea of Biological Species”, en Atran, S. (ed.), Histoire du concept d'espèce dans les sciences de la vie, París: Fondation Singer-Polignac, pp. 101-140.

Toal, C. (2012), "Preaching at the British Association for the Advancement of Science: Sermons, Secularization and the Rhetoric of Conflict in the 1870s", The British Journal for the History of Science 45(1): 75-95.

Tomkins, S. (1963), "Left and Right: A Basic Dimension of Ideology and Personality", en Bruner, K.F. y R.W. White (eds.), The Study of Lives: Essays on Personality in Honor of Henry A. Murray, New York: Atherton Press, pp. 388-411.

Topham, J.R. (2010), "Natural Theology and the Sciences", en Harrison, P. (ed.), The Cambridge Companion to Science and Religion, Cambridge: Cambridge University Press, pp. 59-79.

Turner, F.M. (1974), Between Science and Religion: The Reaction to Scientific Naturalism in Late Victorian England, New Haven: Yale University Press.

van Inwagen, P. y M. Sullivan (2015), "Metaphysics", en Zalta, E.N. (ed.), The Stanford Encyclopedia of Philosophy, URL= http://plato.stanford.edu/archives/spr2015/entries/metaphysics/.

White, P. (2003), Thomas Huxley: Making the "Man of Science", Cambridge: Cambridge University Press.

Yeo, R. (1979), "William Whewell, Natural Theology and the Philosophy of Science in Mid Nineteenth Century Britain", Annals of Science 36(5): 493-516.

Yeo, R. (2003), Defining Science: William Whewell, Natural Knowledge and Public Debate in Early Victorian Britain, Cambridge: Cambridge University Press. 